Author accepted version. Final publication as: Naderi, V., Farsadizadeh, D., Lin, C. and Gaskin, S.J. (2019) A 3D study of an air core vortex using HSPIV and flow visualization, Arabian Journal for Science and Engineering. doi:10.1007/s13369-019-03764-3

\title{
A 3D study of an air-core vortex using HSPIV \& flow visualization
}

\begin{abstract}
A free surface vortex is a mass of water rotating around an axis perpendicular to the free surface. It can occur when withdrawing water from reservoirs or rivers at hydropower intakes with low submergence. Existing vortex models provide general information about the symmetric vortex structure. The vortex structure occurring in an approach flow at the critical submergence condition is examined in detail. In the laboratory, a steady air-core vortex over a bottom intake was created in a wide recirculating flume, in which the water depth, mean velocity of the approach flow and intake discharge could be adjusted. Flow visualization shows that the approach flow results in a non-symmetric velocity distribution in the vortex throughout the flow depth. The detailed set of high-speed particle image velocimetry (HSPIV) data in a series of horizontal and vertical planes was used to observe the formation and evolution of the three-dimensional flow structure of the strong air-core vortex and determine the origin of the vortex. Analysis of this data revealed a complex three-dimensional vortex structure due to the approach flow interacting with the air-core vortex forming a secondary vortex originating at the mixing zone upstream of the vortex, identified by a zero downstream velocity component, and feeding into the upstream side of the intake.
\end{abstract}

Keywords: Vortex structure, asymmetric vortex, bottom intake, HSPIV, flow visualization, vortex origin.

\section{Introduction}

Hydropower plants withdraw water from reservoirs and rivers, which at low submergence can result in the formation of free surface vortices. These can cause operational problems due to air entrainment and non-symmetric inflows resulting in vibrations and reduced performance levels. Many studies have investigated the risk of air entrainment as a function of the flow parameter of critical submergence. Critical submergence is defined as the submergence, or minimum vertical distance between the intake and the free surface, at which air entrainment by the vortex can be clearly observed. As the strength of a free surface vortex increases, it evolves from small coherent surface swirling motions into a fully developed air-core vortex. Different stages of development of an air entraining vortex were described by Denny [1] and the commonly used classification system developed later by Hecker [2] is shown in Fig. 1.

Experimental and analytical investigations of critical submergence have considered the effect of flow conditions such as the angle of the approach flow, the geometry of the intake mouth (mouth shape and intake cross section), location of the intake in the flow and the effect of antivortex devices to reduce critical submergence (e.g., Anwar et al. [3]; Gulliver and Rindels [4]; Hite and Mih [5]; Jain et al. [6]; Borgei and Kabiri-Samani [7]; Naderi et al. [8, 9]; Tastan [10]; Shemshi and Kabiri-Samani [11]; Tastan and Yildirim [12]; and Gao et al. [13]). These applied studies of critical submergence were preceded by investigations of the hydraulic characteristics of vortices. In 1858, Rankine developed a model of a free surface vortex [14], whose tangential velocity is:

$V_{\theta}=\left\{\begin{array}{l}\frac{\Gamma r}{2 \pi r_{m}^{2}}, r \leq r_{m} \\ \frac{\Gamma}{2 \pi r}, r>r_{m}\end{array}\right.$

Where $V_{\theta}$ is the tangential velocity, $r_{m}$ is the radius of the maximum tangential velocity and $\Gamma$ is the maximum circulation of the vortex. Burgers [15] and Rott [16] modelled a stable vortex, having constant vorticity profiles along its axis, by balancing axial stretching with viscous 
Naderi, V., Farsadizadeh, D., Lin, C. and Gaskin, S.J. (2019) A 3D study of an air core vortex using HSPIV and flow visualization, Arabian Journal for Science and Engineering. doi:10.1007/s13369-019-03764-3

diffusion, assuming a linear increase of the axial velocity from the bed to the free surface. Using the Navier-Stokes equations, the tangential velocity is predicted as:

$V_{\theta}=\frac{\Gamma}{2 \pi r}\left[1-\exp \left(-1.25\left(\frac{r}{r_{m}}\right)^{2}\right)\right]$

Odgaard [17] used this model to predict critical submergence in an air core vortex. Mih [18] modified the Rankine vortex model based on experimental data to predict the tangential velocity as:

$V_{\theta}=\frac{\Gamma}{2 \pi r_{m}} \frac{2\left(\frac{r}{r_{m}}\right)}{1+2\left(\frac{r}{r_{m}}\right)^{2}}$

Wang et al. [19] performed detailed PTV experiments for an intake vortex in a scale model of a dam (in a rectangular channel with an approach flow). Using an empirical equation to provide a best fit for the observed data, the tangential velocity was expressed as

$V_{\theta}=\frac{\Gamma}{2 \pi r_{m}} \frac{m_{1}\left(\frac{r}{r_{m}}\right)}{1+m_{2}\left(\frac{r}{r_{m}}\right)+\left(\frac{r}{r_{m}}\right)^{2}}$

Where $m_{1}=0.928$ and $m_{2}=-0.7$. Studies have also developed models to predict the axial vortex velocity (or vertical velocity of the vortex flow field). Empirical equations to fit observations were used by Hite and Mih [5] and Wang et al. [19] to propose formulas for the vertical velocity as Eqs. (5) and (6), respectively:

$W=\frac{Q z}{4 \pi d r_{m}^{2}} \frac{16}{\left(1+2\left(\frac{r}{r_{m}}\right)^{2}\right)^{2}}$
$W=\frac{Q z}{4 \pi d r_{m}^{2}} \frac{3 \times m_{1}}{\left(1+m_{2}\left(\frac{r}{r_{m}}\right)+\left(\frac{r}{r_{m}}\right)^{\wedge} 2\right)^{2}}$

Where, $W, Q, z$ and $d$ are, the axial velocity of the vortex, intake discharge, vertical axes (downward positive) and intake diameter, respectively. Experimental studies of the vortex flow field have measured velocity using the point measurement methods of Acoustic Doppler Velocimetry (ADV) or Laser Doppler Anemometry (LDA) [5, 20]. Particle Image Velocimetry (HSPIV) provides two-dimensional velocity fields and allows for the calculation of turbulence statistics such Reynolds stresses [19]. PIV has been used to study the vortex flow field under approach flow conditions for the different vortex types of Hecker (See Fig 1) [21], the time averaged velocity fields of a spiral vortex at a pump-intake [22, 23] and the velocity components close to a horizontal intake with an approach flow [24, 25]. Suerich-Gulick et al. [24] investigated the relationship between the axial vortex velocity and the intake approach flow velocity for a horizontal intake. Furthermore, they proposed a model to estimate the vortex circulation and maximum water surface depression as a function of the approach flow velocity and the relative submergence. Experimental studies were conducted on air-entraining vortices examining the effect of the approach flow geometry on the vortex structure [26, 27]. However, the detailed flow structure of a strong air-core vortex under critical submergence has not yet been explored. A better understanding of this phenomenon is required both for basic research and for applied engineering systems to evaluate the risk of vibrations and the potential magnitude of reduction in performance.

In the present work, a steady air-core vortex was established under the critical submergence condition for a vertical bottom intake located in an approach flow. The vortex structure and velocity fields were observed using HSPIV at horizontal sections at intervals over the depth, and at two vertical sections upstream of the intake. A new method for determining the origin of the vortex was developed. The experimental data allowed for the description of the threedimensional structure of the vortex in an approach flow. In the mixing zone, which forms 
Naderi, V., Farsadizadeh, D., Lin, C. and Gaskin, S.J. (2019) A 3D study of an air core vortex using HSPIV and flow visualization, Arabian Journal for Science and Engineering. doi:10.1007/s13369-019-03764-3

upstream of the vortex, a rotating cell develops aligned with the velocity contour having zero downstream component.

\section{Experimentation}

\subsection{Water Channel and Intake System}

The experiments were performed in a re-circulating water channel $4.95 \mathrm{~m}$ long and $1 \mathrm{~m}$ wide with glass-walls and a Plexiglas-bottom located in the Laser Laboratory of Department of Civil Engineering, National Chung Hsing University, Taiwan (shown in Figure 2). The flow enters the flume from an inlet reservoir $1.55 \mathrm{~m}$ long, $1 \mathrm{~m}$ wide and $1.4 \mathrm{~m}$ deep, in whose lower section a multi-layered and perforated energy dissipating section, made of vertical pipes $5 \mathrm{~mm}$ in diameter and $100 \mathrm{~mm}$ in height, ensured the inflow was uniform and free of circulation. At the downstream end of the flume, a reservoir, $1.55 \mathrm{~m}$ long, $1 \mathrm{~m}$ wide and $1.4 \mathrm{~m}$ deep, collects the flow from where it is re-circulated to the inlet reservoir using a digitally-controlled centrifugal pump. The test section was $2 \mathrm{~m}$ long, $1 \mathrm{~m}$ wide and $0.4 \mathrm{~m}$ high. A steady air-core vortex was created at a bottom intake system by modifying the submergence using a $2 \mathrm{~m}$ long, $1 \mathrm{~m}$ wide and $10 \mathrm{~mm}$ thick streamlined horizontal plate placed parallel to the flume bed whose vertical elevation (in the range of 10 to $100 \mathrm{~mm}$ ) could be adjusted. The vertical intake pipe (ID 94 $\mathrm{mm}$ ) was installed flush with the top surface of the adjustable submergence plate on its midline and $1.25 \mathrm{~m}$ from its leading edge and passed through the bottom Plexiglas of the flume. The intake discharge, which was set with a digitally-controlled centrifugal pump, was returned to the downstream reservoir. The formation of a strong air-core vortex was promoted by enhancing the asymmetry of the approach flow with an offset vertical plate $(1.75 \mathrm{~m}$ long, 0.5 $\mathrm{cm}$ high and $10 \mathrm{~mm}$ thick) placed parallel and at a distance of $208 \mathrm{~mm}$ from the downstream left wall. Therefore, the intake was located $282 \mathrm{~mm}$ from the left plate and $500 \mathrm{~mm}$ from the right-side wall (see Figs. 2 and 4). In the current study, the approach flow depth over the horizontal plate was $118 \mathrm{~mm}$, the mean approach flow velocity was $66 \mathrm{~mm} / \mathrm{s}$, and the bottom intake discharge was $4.61 \mathrm{l} / \mathrm{s}$. These conditions produced a strong, stable free-surface vortex with an air core, whose critical submergence was1 $18 \mathrm{~mm}$.

\subsection{HSPIV and Flow Visualization Setup}

Flow visualization was accomplished by illuminating $2 \mathrm{~mm}$ light planes with a continuous Innova 300C Argon Ion laser. A composite horizontal cross-section (see Figs 3(a) and 4) was formed from eight fields of view (FOVs) recorded with a high-speed CCD camera perpendicular to the laser sheet. The high-speed camera, a Phantom Miro eX4, with an $800 \mathrm{x}$ 600 pixels resolution equipped with a Nikon $50 \mathrm{~mm}$ camera lens, was synchronized with the laser for pulses at $100 \mathrm{fps}$ with an exposure time of $10 \mathrm{~ms}$. The total acquisition time for each test was $42 \mathrm{~s}$ corresponding to 4411 images. The camera was placed under the flume for horizontal sections and on the right side of the flume for the vertical sections. The tracer particles for the HSPIV were aluminium powder having a purity of $99 \%$. Horizontal sections were obtained at elevations of $0.25 h, 0.5 h$, and $0.75 h$, where $\mathrm{h}$ is the water depth in the flume. Two vertical sections were obtained along the centreline of the bottom intake, the first immediately upstream of the upstream edge of the bottom intake and the other $0.5 \mathrm{~m}$ upstream (see Fig 3(b)).

The origin of the coordinate system describing the vortex should be located at its origin. However, during preliminary tests it was observed that the origin of the air-core vortex did not 
Naderi, V., Farsadizadeh, D., Lin, C. and Gaskin, S.J. (2019) A 3D study of an air core vortex using HSPIV and flow visualization, Arabian Journal for Science and Engineering. doi:10.1007/s13369-019-03764-3

coincide with the origin of the bottom intake due to the unsteady nature of the vortex. Therefore, the origin used for the HSPIV measurement was taken as the centre of the intake pipe. Furthermore, based on the calculated HSPIV data, the real origin of the vortex was obtained using an iterative method. In the coordinate system used, the $X$-axis, is horizontal and positive in the streamwise direction of the flow, the $Y$-axis is horizontal and normal to the $X$ axis (positive to the downstream left), and the vertical axis, $Z$, is oriented upwards (conforming to the right-hand rule). A cylindrical coordinate system is also used, in which in the horizontal plane the radial velocity $V_{r}$ is outwards and the tangential velocity $V_{\theta}$ is perpendicular to the radial velocity (anticlockwise) and tangent to the velocity of the rotating fluid. The vortex rotation in a counter-clockwise direction is positive. The location of the eight fields of view that make up the composite horizontal images is shown in Fig. 4.

\section{Experimental Results and discussion}

\subsection{Determination of the origin of the vortex flow}

Due to the unsteady nature of the vortex flow and the influence of the approach flow, the origin of the vortex does not necessarily coincide with the center of the intake pipe, which, although typically assumed in previous work, may lead to inaccuracies. A top view of the present aircore vortex, captured at different time steps from a fixed camera view, demonstrates the unsteady nature of the vortex (see Fig 5). The time averaged horizontal velocity field of the vortex at $Z=0.5 h$, determined from the horizontal HSPIV data, is shown in Fig. 6a, in which the magnitude of the arrows represents the velocity magnitude. This is compared to the theoretical velocity distribution, in Fig. 6b, determined by adding the approach flow velocity to the tangential vortex velocity from the Burgers/Rott model $[15,16]$. Highlighted in yellow in both figures is the mixing line, define as the line at which the downstream velocity component is zero, which is discussed further in later sections.

Analysis of the horizontal velocity fields of the vortex, obtained from the eight different FOVs, requires the accurate determination of the origin for determination of the circulation, and the tangential and radial velocities in a cylindrical coordinate system. A method was developed to determine the origin by plotting the time averaged velocity components $(U, V)$ of the vortex flow in a Cartesian coordinate system. Each horizontal plane of the velocity field (HSPIV data) is divided into quadrants as shown in Fig.7. The sign and magnitude of the $U$ and $V$ velocity components of the tangential velocity is summarized as follows: Quadrant 1: $U$ is negative, and $V$ is positive; Quadrant 2: $U$ and $V$ are negative; Quadrant 3: $U$ is positive, and $V$ is negative, and Quadrant 4: $U$ and $V$ are positive. The property, that the maximum $U$ or $V$ component should be located at the radial position of $0^{\circ}, 90^{\circ}, 180^{\circ}$ and $270^{\circ}$ corresponding to $\mathrm{P} 1, \mathrm{P} 2, \mathrm{P} 3$ and $\mathrm{P} 4$ respectively, is used to determine the origin. The horizontal location of the maximum magnitude of the $U$ component of velocity indicates the location of P2 $\left(-U_{\max }\right)$ and P4 $\left(+U_{\max }\right)$ and hence the shift in the horizontal location of the origin is by $\Delta x=0.9 \mathrm{~cm}$ (see Fig. 8a) for the data at $Z=0.5 \mathrm{~h}$. Similarly, the horizontal location of the maximum magnitude of the $\mathrm{V}$ component of velocity is indicated by the location of $\mathrm{P} 1\left(+V_{\max }\right)$ and $\mathrm{P} 3\left(-V_{\max }\right)$ and hence the shift in the horizontal location of the origin is by $\Delta y=1.3 \mathrm{~cm}$ (see Fig. $8 \mathrm{~b}$ ). This is compared to the result from Burgers/Rott theoretical model $[15,16]$ of the tangential vortex velocity added to the approach flow velocity, see Fig. 8c and 8d. The differences observed are due to the theoretical model being a 2-D representation, which does not capture the $3 \mathrm{D}$ effects of the interaction between the approach flow and the vortex flow described below.

The effect of the approach flow on the vortex is seen both in the shift in the location of the origin of the vortex flow $(\Delta x=0.9 \mathrm{~cm}, \Delta y=1.3 \mathrm{~cm})$ and in the non-symmetrical distribution 
Naderi, V., Farsadizadeh, D., Lin, C. and Gaskin, S.J. (2019) A 3D study of an air core vortex using HSPIV and flow visualization, Arabian Journal for Science and Engineering. doi:10.1007/s13369-019-03764-3

of the $U$ velocity component as seen in Fig. 8(a) (at P2, $U=37 \mathrm{~cm} / \mathrm{s}$ and at $\mathrm{P} 4, U=-32 \mathrm{~cm} / \mathrm{s}$ ). This will be discussed further below.

\subsection{Flow visualization}

The 3D structure of a vortex under an approach flow and at the critical submergence condition is visualized to elucidate the effect of the approach flow on the flow structure of an air-core vortex and the flow field directly upstream. A schematic view of the flow structure of the mixing zone due to the interaction of the vortex and approach flows is shown in Fig 9. The flow follows descending helicoidal path-lines around the core of the air-core vortex. Upstream of the vortex, the approach flow approaches the vortex and on meeting the tangential velocity of the vortex flow in the upstream direction decelerates creating a turbulent mixing zone and is entrained into a secondary vortex. The mixing and entrainment zone is centered on the line along which the approach flow velocity in the streamwise direction is reduced to zero. As the vortex rotates in a counter-clockwise direction, the mixing line is located on the left side of flume where the approach flow and tangential velocity are in opposing directions. As shown in Fig 9, the steady air-core vortex flow obstructs the approach flow (similarly to a pier in river flow), causing entrainment into a long curved secondary vortex forming in the mixing zone spanning half the flow width (about the line $\mathrm{y}=-\mathrm{x}$ for $\mathrm{x}>0$ ). The approach flow entrained in the mixing zone, therefore, moves along a helicoidal path, starting from the left side of the flume upstream of the air-core vortex and spiralling in to join the air-core vortex near its base in the middle of the flume (where the vortex flow does not have a flow component opposing the approach flow). This helicoidal flow from the secondary vortex results in a reduced flow velocity above the secondary vortex and an increased velocity below the secondary vortex (due to addition of the secondary vortex flow and approach flow velocities). The exact location of the secondary vortex varies over time as it is unsteady. Note that the Burgers/Rott model $[15,16]$ does not capture this 3D flow behaviour. The flow visualization technique used in both horizontal and vertical planes revealed more information about the flow dynamics of the mixing zone and its effect on the vortex structure.

In previous studies on the vortex structure, based on the symmetric Rankine vortex model, it is assumed that the fluid moves in a closed entirely symmetric circular paths in any horizontal plane. However, when an approach flow is present, it causes the flow's trajectory to be asymmetric throughout the depth. Fig 10 shows two high-resolution streak-line images, captured using the flow visualization technique, of the FOVs \#1 and \#2 at a depth of $Z=0.75 \mathrm{~h}$. (Note the overlap of 30\% in the field of view for adjacent FOVs, seen in Figures 4 and 10). The length of the streak-lines indicates the velocity magnitude. As seen in plan-view, as the approach flow and the tangential velocity of the air-core vortex approach each other in the mixing zone (FOVs \#1 and \#2), both velocities start to decrease and reach zero along the line shown in white in Fig 10. The evolution of the mixing region can also be seen in the intake midline vertical sections ( 3 images at $4 \mathrm{~s}$ intervals at $Y=0$,) in Fig. 11. For the vertical FOV aligned with the intake, $Y=0 \mathrm{~cm}$, a small horizontal eddy ( $Y$ vorticity) was observed for $t=$ $0 s$, in which the approach flow was dominant, as shown by the longer streak-lines (Fig 11(a)). In Fig 11(b), 4 seconds later, the initial structure breaks down into a vortex pair consisting of two small horizontal vortices with opposite-signed vorticity, and the approach flow divides flowing above and below the vortex pair. By $t=8 \mathrm{~s}$, the vortex pair joins again into a small coherent eddy ( $Y$ vorticity) (Fig 11(c)). To summarize, the vertical flow visualization images at $Y=0 \mathrm{~cm}$ illustrate the high velocity of the flow below the secondary vortex where the approach flow and horizontal vortex flows are coincident. The secondary vortex rotating core is unsteady varying in strength and location (in X and Z-directions). This flow pattern is confirmed in the time-averaged HSPIV measurements in Fig 15. 
Naderi, V., Farsadizadeh, D., Lin, C. and Gaskin, S.J. (2019) A 3D study of an air core vortex using HSPIV and flow visualization, Arabian Journal for Science and Engineering. doi:10.1007/s13369-019-03764-3

The secondary vortex shown in Fig 11 has a significant effect on the downstream $(U)$ velocity distribution. This effect, which we call "the secondary vortex rotational cell effect", divides the approach flow into a low velocity upper flow and a high velocity lower flow.

\subsection{Tangential velocity distribution}

Horizontal sections of the vortex showing the time-averaged velocity vector fields calculated using HSPIV were obtained from a composite of the 8 FOVs defined in Fig. 4. The true origin of the vortex was determined at each elevation, to allow for the determination of the tangential velocity distribution along radial profiles. In Fig. 12, the tangential velocity along radial profiles at $0^{\circ}, 90^{\circ}, 180^{\circ}$, and $270^{\circ}$ are plotted showing maximums of $28.9 \mathrm{~cm} / \mathrm{s}, 24.7 \mathrm{~cm} / \mathrm{s}, 24.8$ $\mathrm{cm} / \mathrm{s}$ and $30.1 \mathrm{~cm} / \mathrm{s}$ at a radius of $7.1 \mathrm{~cm}, 6.4 \mathrm{~cm}, 7.6 \mathrm{~cm}$ and $7.4 \mathrm{~cm}$, respectively. The effect of the approach flow on the vortex structure is also visible in the variation of the velocity with angular position. The maximum tangential velocity was observed at P4 $\left(270^{\circ}\right)$, where the approach flow was aligned with the vortex flow. The variation in the average tangential velocity distribution (at angular intervals of $22.5^{\circ}$ averaged between $5 \mathrm{~cm} \leq r \leq 10 \mathrm{~cm}$ at $Z=0.75 h$ ) is shown in Fig 13. The maximum tangential velocities are observed in quadrant 3, while the minimums are observed in quadrant 2 , where a component of the tangential velocity opposes the approach flow direction. The observations of the velocity field were the same for $Z=0.25 h$ and $Z=0.5 h$.

In summary, the observations show the asymmetric structure of the air-core vortex due to the effect of the approach flow. The mixing zone producing the secondary vortex is recognized as the region upstream of the vortex core in which streamwise component of velocity is zero (See yellow highlight in Fig 6). The symmetric models of Rankine (1858), Odgard (1986) and Mih (1990) are approximations of the air-core vortex in an approach flow, as they do not describe the asymmetry [14, 17 and 18].

A vortex can be characterized by its circulation, which is a macroscopic measure of rotation for a finite area of a fluid. By assuming a given closed curve (or a vortex streamline), the circulation is obtained by integrating the tangential velocity, $V_{\theta}$, along this curve. The magnitude of the circulation is a measure of the vortex strength and is related to the vorticity component orthogonal to the vertical. Vortex circulation can be calculated using Eq. (7) [17].

$\Gamma=\oint_{C} \vec{v} \overrightarrow{d l}=\iint_{S} \vec{w} \overrightarrow{d s}$

Where $\boldsymbol{\Gamma}$ is circulation, $\overrightarrow{\boldsymbol{v}}$ is the flow velocity, $\mathrm{S}$ is an arbitrary surface bounded by a curve of $C$ and $\overrightarrow{\boldsymbol{w}}$ is vorticity. The vorticity of a flow is defined as the curl of the velocity field and is defined as:

$\vec{w}=\nabla \times \vec{v}$

The bulk circulation is defined as the overall circulation of the total mass of the vortex and can be obtained by finding the average circulation far from the vortex core. In many previous studies, the vortex circulation was imposed by the geometry of the flow configuration. The bulk circulation for the present study of $1584 \mathrm{~cm}^{2} / \mathrm{s}$ is used to non-dimensionalize the data. The radial profiles of the normalized averaged tangential velocity are compared to the models of Rankine [14], Burgers/Rott [15/16] and Mih [18] in Fig. 14 [14, 17 and 18]. As also reported by Suerich-Gulik et al. [24, 25], the Rankine [14] model overestimates the peak tangential velocity but the profile follows a similar shape. The profile shape predicted by the Burgers/Rott [15/16] and Mih [18] models are similar in shape but have a higher peak value and lower values 
Naderi, V., Farsadizadeh, D., Lin, C. and Gaskin, S.J. (2019) A 3D study of an air core vortex using HSPIV and flow visualization, Arabian Journal for Science and Engineering. doi:10.1007/s13369-019-03764-3

for $\boldsymbol{R}=\frac{r}{r_{m}} \geq 1.5$. This underestimation is due to the effect of the mixing zone in Quadrant 2 on the overall averaged velocity values of the air-core vortex. Therefore, the model fits in quadrants 1,3 and 4 but not in quadrant 2 .

The average over all depths of the average normalized tangential velocity profiles match the shape of the profile (similar peak values) found by Wang et al. [19], who also studied a vortex in an approach flow using particle tracking velocimetry.

\subsection{Axial velocity distribution}

The time-averaged HSPIV velocity fields for two vertical FOVs are shown in Fig 15. The instantaneous streak-lines seen in the vertical section at $Y=0 \mathrm{~cm}$ in Fig 11, illustrate a recirculation cell with $-Y$ (negative $Y$ ) rotation about a span-wise axis located approximately at mid-depth. This results in a sweep of flow below the recirculating cell due to the alignment of the velocity from the secondary vortex and that of the air-core vortex and a reduction in the flow velocities above the cell as these velocities are in opposing directions. In the timeaveraged HSPIV velocity vector field at $Y=0 \mathrm{~cm}$ (Fig 15 (b)), a high-velocity region is seen at lower depths and, at higher elevations, a stagnation region is seen upstream of the air-core vortex. The undisturbed approach flow can be seen in Fig. 15(a) $0.5 \mathrm{~m}$ upstream of the air-core vortex. Based on the flow visualization figures reported in Fig 11 and on the time averaged data of Fig. 15(b), an unsteady rotational cell of the secondary vortex occurs in the mixing zone, which, over time, can be found between $X=[-3,-8]$ and between $Z=[5,12]$ but is most frequently centred on $(X, Z)=(-6,10)$. The streamwise $(U)$ and axial $(W)$ velocity magnitudes are clearly seen in the vertical profiles shown in Fig 16. A steady constant horizontal approach flow velocity is seen far upstream of the intake. Closer to the intake, at $X=-14.7 \mathrm{~cm}$, the flow splits to flow over and below the secondary vortex vortex (rotational cell effect) and by $X=-$ $12.3 \mathrm{~cm}$, the flow is downward and toward the air-core vortex. At $X=-7 \mathrm{~cm}$ and $X=-5 \mathrm{~cm}$, the streamwise velocity stagnates at higher elevations while there is a strong downwards streamwise sweep of flow into the air-core vortex near its base.

The evolution with downstream position of the streamwise $(U)$ and vertical $(W)$ velocity from the horizontal profiles is presented in Fig. 17. Again, the rotational cell of the secondary vortex can be identified in the profiles at $Z=8 \mathrm{~cm}$ and $Z=10 \mathrm{~cm}$ as the velocity magnitude decreases to zero at the vortex. Note also that the vertical velocity at $Z=8 \mathrm{~cm}$ is upwards for $X \leq-14 \mathrm{~cm}$ and decreases gradually to become zero at $X=-50 \mathrm{~cm}$.

The axial velocity best-fit equations for a symmetric vortex by Hite and Mih [5] and a vortex in an approach flow by Wang et al. [19] were compared to the experimental results. These equations describe the evolution of the velocity profile over the depth as they are a function of radius and water depth. Fig 18 presents the results of the axial velocity equations as a function of radius and compares them to the experimental results at $Z=0.25 h, Z=0.5 h$, and $Z=0.75 h$. The non-dimensional axial velocity, $W_{N}$, is defined as Eq. (9):

$W_{N}=\frac{4 \pi d r_{m}^{2}}{Q} \frac{W}{z}$

where all the parameters have been defined previously (recall $W$ is positive upwards).

The comparison axial velocity of the symmetric vortex of the models to the approach flow vortex of the experimental data shows the effect of the recirculating cell of the secondary vortex 
on the axial velocity. The recirculating cell of the secondary vortex, located just upstream of the air-core vortex, blocks and entrains the approach flow and modifies the axial velocities. The secondary vortex is unsteady, and its position varies from $Z=4$ to $12 \mathrm{~cm}$. At $Z=0.25 \mathrm{~h}$, the velocities of the secondary vortex are aligned with the intake vortex flow so there is a good match with the symmetric vortex model. While at $Z=0.5 h$ and $Z=0.75 h$, due to the presence of the secondary vortex, the velocities are aligned for $R>1.3$ and opposing for $R<1.3$. The difference from the model increases with depth as the secondary vortex is more often at the higher elevations.

\section{Conclusion}

A steady air-core vortex was created in a wide flume with an approach flow and under critical submergence conditions. Particle image velocimetry and flow visualisation techniques were used to study the air-core vortex structure. By using horizontal velocity vector fields from PIV, a new method for determining the accurate origin of the vortex at each elevation was introduced. The horizontal and vertical PIV sections of the vortex flow allowed its detailed three-dimensional structure to be observed. The obstruction and entrainment of the approach flow by the strong air-core vortex results in a mixing zone characterized by a secondary vortex spanning half the flume width and extending from high on left wall high to the base of the air core vortex. The mixing zone is identified by the line along which the interaction of the approach flow velocity and the tangential vortex velocity resulted in a zero-downstream velocity component. Flow paths in the mixing zone, therefore, follow a helicoidal path around this secondary vortex line joining the strong air-core vortex at its base. Differences in the velocity profiles of the strong air-core vortex in an approach flow to those of a symmetric vortex modelled (or represented by empirical equations) are due to the rotating cell of the secondary vortex.

\section{REFERENCES}

1- Denny, D. F.: An experimental study of air-entraining vortices in pump sumps. Proceedings of the Institution of Mechanical Engineers, London, England. 170(1), 106-125 (1956)

2- Hecker, G.E.: Fundamentals of vortex intake flow. Conclusions. In: Knauss. Swirling flow problems at intakes. IAHR hydraulic structures design manual. Rotterdam: Balkema, [chapters 2 and 8]. (1987)

3- Anwar, H.O., Weller, J.A., and Amphlett, M.B.: Similarity of free-vortex at horizontal intake. Journal of Hydraulic Research. 16(2), 95-105 (1987)

4- Gulliver, J.S., and Rindels, A.J.: Weak vortices at vertical intakes. Journal of Hydraulic Engineering, ASCE. 113(9), 1101-1116 (1987)

5- Hite, J.E., and Mih, W.C.: Velocity of air-core vortices at hydraulic intakes. Journal of Hydraulic Engineering, ASCE. 120(3), 284-297 (1994)

6- Jain, A.K., Rangaraju, K.G., and Garde, R.J.: Vortex formation at vertical pipe intakes. Journal of the Hydraulics Division. ASCE. 104(10), 1429-1448 (1978)

7- Borghei, S.M., and Kabiri-Samani, A.R.: Effect of anti-vortex plates on critical submergence at a vertical intake. Scientia Iranica. 17(2), 89-95 (2010)

8- Naderi, V., Farsadizadeh, D., Hosseinzadeh-Dalir, A., and Arvanaghi, H.: Experimental study of bellmouth intakes on discharge coefficient". Journal of Civil Engineering and Urbanism. 3(6), 368-371 (2013)

9- Naderi, V., Farsadizadeh, D., Hosseinzadeh-Dalir, A., and Arvanaghi, H.: Effect of using vertical plates on vertical intake on discharge coefficient. Arabian Journal for Science and Engineering. 39(12), 86278633 (2014)

10- Tastan, K.: Scale and flow boundary effects for air-entraining vortices. Water Management. 170(4):1-9 (2016)

11- Shemshi, R., and Kabiri-Samani, A.: Swirling flow at vertical shaft spillways with circular piano-key inlets. Journal of Hydraulic Research. 55(2), 248-258 (2017) 
Naderi, V., Farsadizadeh, D., Lin, C. and Gaskin, S.J. (2019) A 3D study of an air core vortex using HSPIV and flow visualization, Arabian Journal for Science and Engineering. doi:10.1007/s13369-019-03764-3

12- Tastan, K., and Yildirim, N.: Effects of Intake Geometry on the Occurrence of a Free-Surface Vortex. Journal of Hydraulic Engineering. 144(4):04018009 (2018)

13- Gao, X., Zhang, H., Liu, J., Sun, B., and Tian, Y.: Numerical investigation of flow in a vertical pipe inlet/outlet with a horizontal anti-vortex plate: effect of diversion orifices height and divergence angle. Engineering Applications of Computational Fluid Mechanics. 12(1):182-194 (2018)

14- Rankine, W.J.M.: A Manual of Applied Mechanics, Charles Griffin, London. (1858)

15- Burgers, J.M.: A mathematical model illustrating the theory of turbulence. Advances in Applied Mechanics. 1, 171-199 (1948)

16- Rott, N.: On the viscous core of a line vortex. Z. Angew. Math. Phys. 9b, 543-553 (1958)

17- Odgaard, A.J.: Free-surface air core vortex. Journal of Hydraulic Engineering. ASCE. 112(7), 610-620 (1986)

18- Mih, W.C.: Analysis of fine particle concentrations in a combined vortex. Journal of Hydraulic Research. 28(3), 392-39 (1990)

19- Wang, Y.K., Jiang, C.B., and Liang, D.F.: Comparison between empirical formulae of intake vortices. Journal of Hydraulic Research. 49(1), 113-116 (2011)

20- Sarkardeh, H., Zarrati, A.R., and Roshan, R.: Effect of intake head wall and trash rack on vortices. Journal of Hydraulic Research. 48(1), 108-112 (2010)

21- Li, H., Chen, H., Zheng, M.A., and Zhou, Y.: Experimental and numerical investigation of free surface vortex. Journal of Hydrodynamics. 20(4), 485-491 (2008)

22- Rajendran, V.P. and Patel, V.C.: Measurement of vortices in model pump-intake bay by PIV. Journal of Hydraulic Engineering, ASCE. 126(5), 322-334 (2000)

23- Okamura, T., Kamemoto, K., and Matsui, J.: CFD prediction and model experiment on suction vortices in pump sump. Proceedings of 9th Asian International Conference on Fluid Machinery, October, Jeju, South Korea, AICFM9-053 (2007)

24- Suerich-Gulick, F., Gaskin, S.J., Villeneuve, M., and Parkinson, E.: Characteristics of Free Surface Vortices at Low Head Hydropower Intakes. Journal of Hydraulic Research. 140, 291-299 (2014a)

25- Suerich-Gulick, F., Gaskin, S.J., Villeneuve, M., and Parkinson, E.: Free surface intake vortices: theoretical model and measurements. Journal of Hydraulic Research. 52(4), 502-512 (2014b)

26- Möller, G. Vortex-induced air entrainment rate at intakes. Diss., Eidgenössische Technische Hochschule ETH Zürich. Nr. 21277 (2013)

27- Mulligan S., Casserly J., Sherlock R.: Experimental and Numerical Modelling of Free-Surface Turbulent Flows in Full Air-Core Water Vortices. In: Gourbesville P., Cunge J., Caignaert G. (eds) Advances in Hydroinformatics. Springer Water. Springer, Singapore. 549-569 (2016)

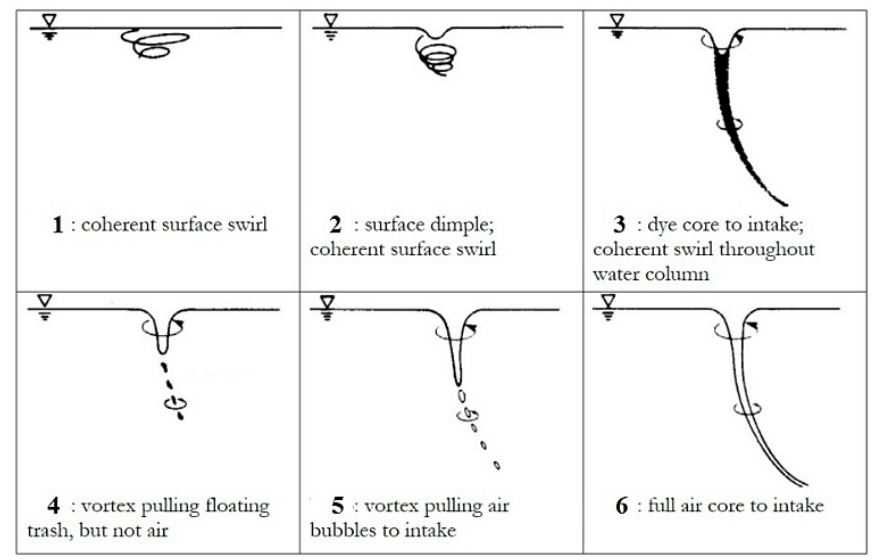

Figure 1. Vortex type classification (Hecker 1987) [2] 
Naderi, V., Farsadizadeh, D., Lin, C. and Gaskin, S.J. (2019) A 3D study of an air core vortex using HSPIV and flow visualization, Arabian Journal for Science and Engineering. doi:10.1007/s13369-019-03764-3
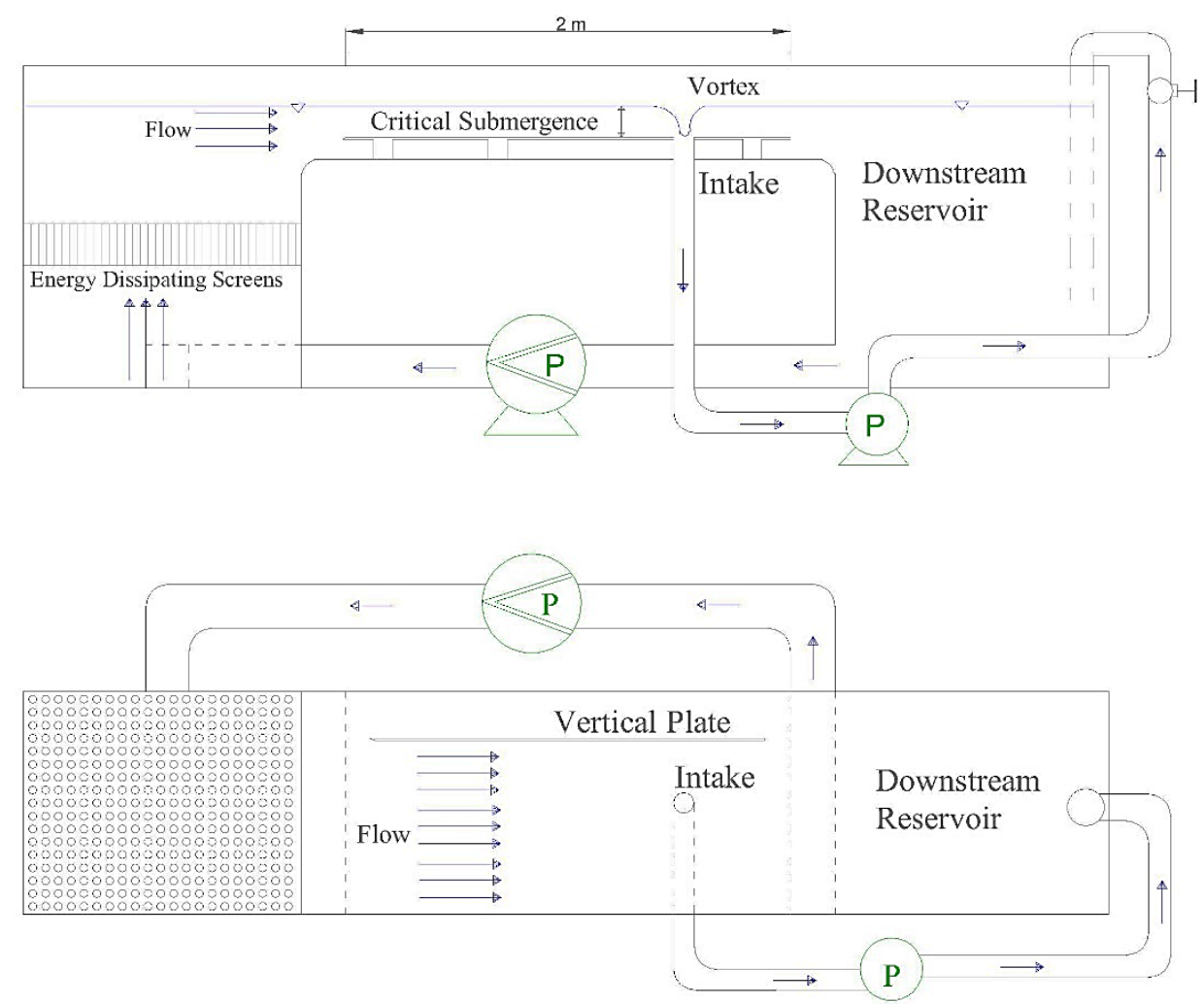

Figure 2- Schematic diagram of experimental setup: (a) side view and (b) top view

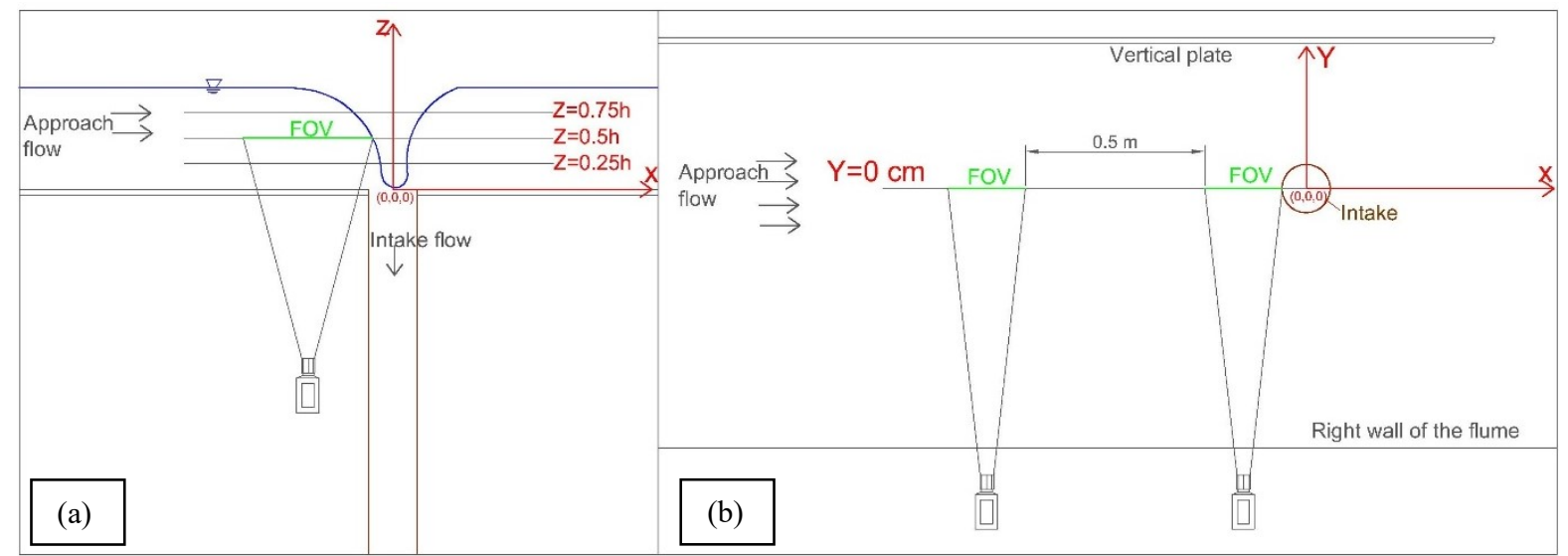

Figure 3- Schematic of HSPIV and flow visualization setup; (a): Horizontal PIV images (side view of the flume); (b) Vertical PIV images (plan view of the flume) 
Naderi, V., Farsadizadeh, D., Lin, C. and Gaskin, S.J. (2019) A 3D study of an air core vortex using HSPIV and flow visualization, Arabian Journal for Science and Engineering. doi:10.1007/s13369-019-03764-3

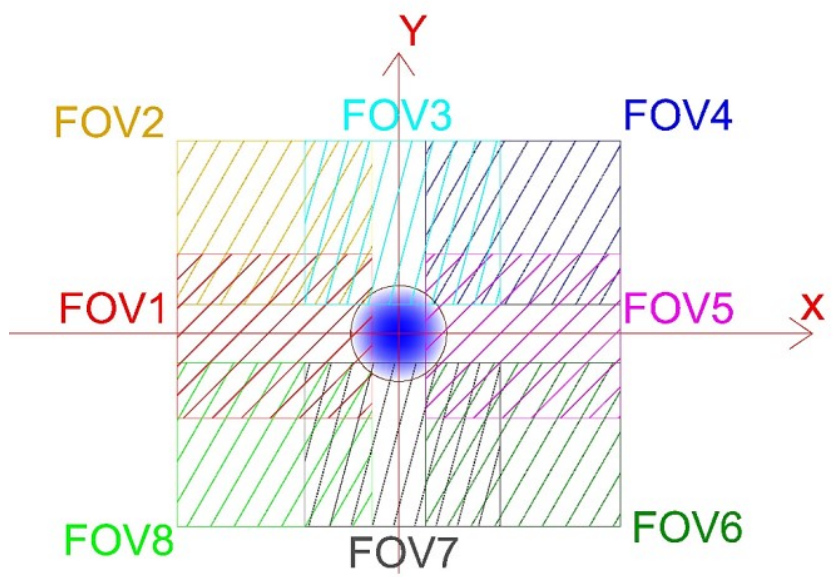

Figure 4- The 8 FOVs at each horizontal HSPIV elevation (plan view).

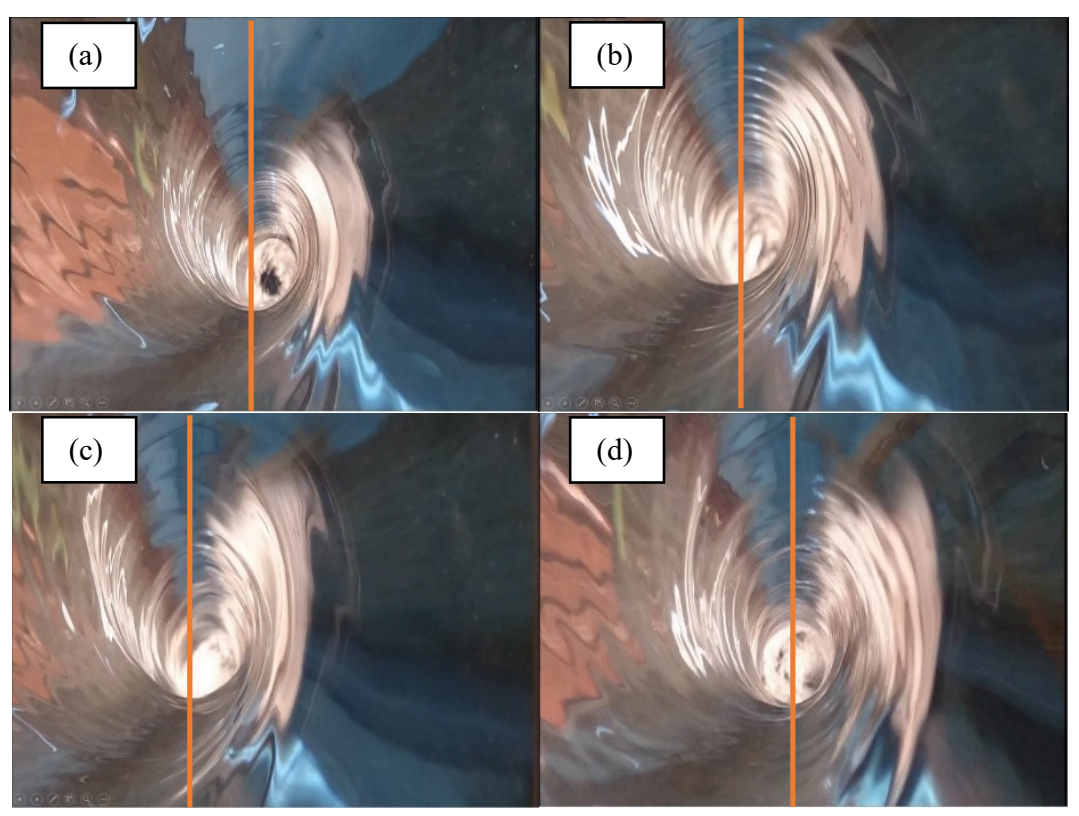

Figure 5- Top view of the strong air-core vortex from a fixed field of view captured at a) $t=1 \mathrm{~s}, \mathrm{~b}) t=2 \mathrm{~s}$, c) $t=3 \mathrm{~s}$ and d) $t=4 \mathrm{~s}$ (Vertical orange lines are the approximations of the origin of the vortex on $X$-axis) 
Naderi, V., Farsadizadeh, D., Lin, C. and Gaskin, S.J. (2019) A 3D study of an air core vortex using HSPIV and flow visualization, Arabian Journal for Science and Engineering. doi:10.1007/s13369-019-03764-3

(a)

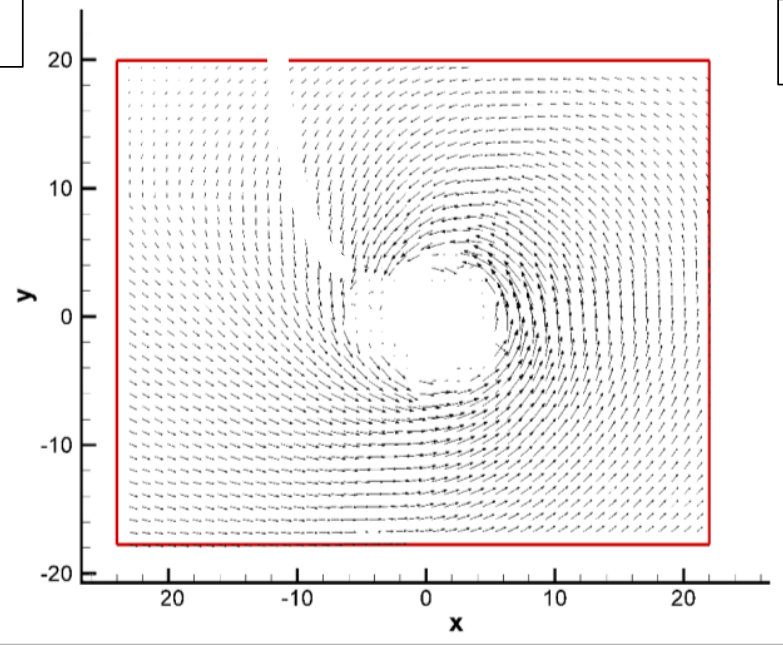

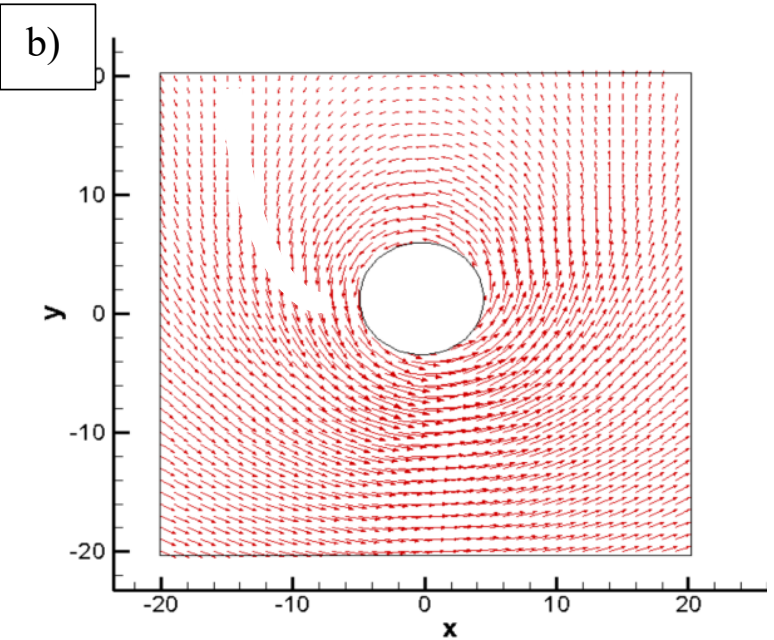

Figure 6-a) Experimental data of the time-averaged velocity vector field of horizontal HSPIV at $Z=0.5 h$. b) The approach flow velocity added to Burgers/Rott model for the tangential vortex velocity. The mixing line is highlighted in yellow.

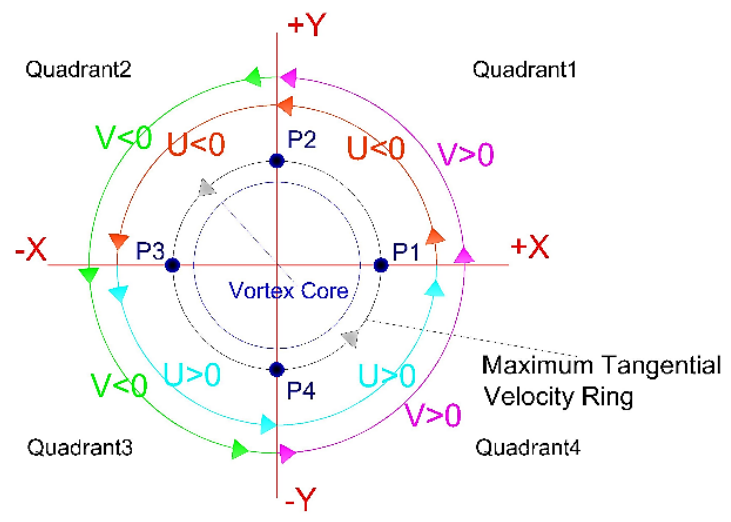

Figure 7- Schematic plan of cross-section of the vortex divided into Cartesian quadrants 
Naderi, V., Farsadizadeh, D., Lin, C. and Gaskin, S.J. (2019) A 3D study of an air core vortex using HSPIV and flow visualization, Arabian Journal for Science and Engineering. doi:10.1007/s13369-019-03764-3
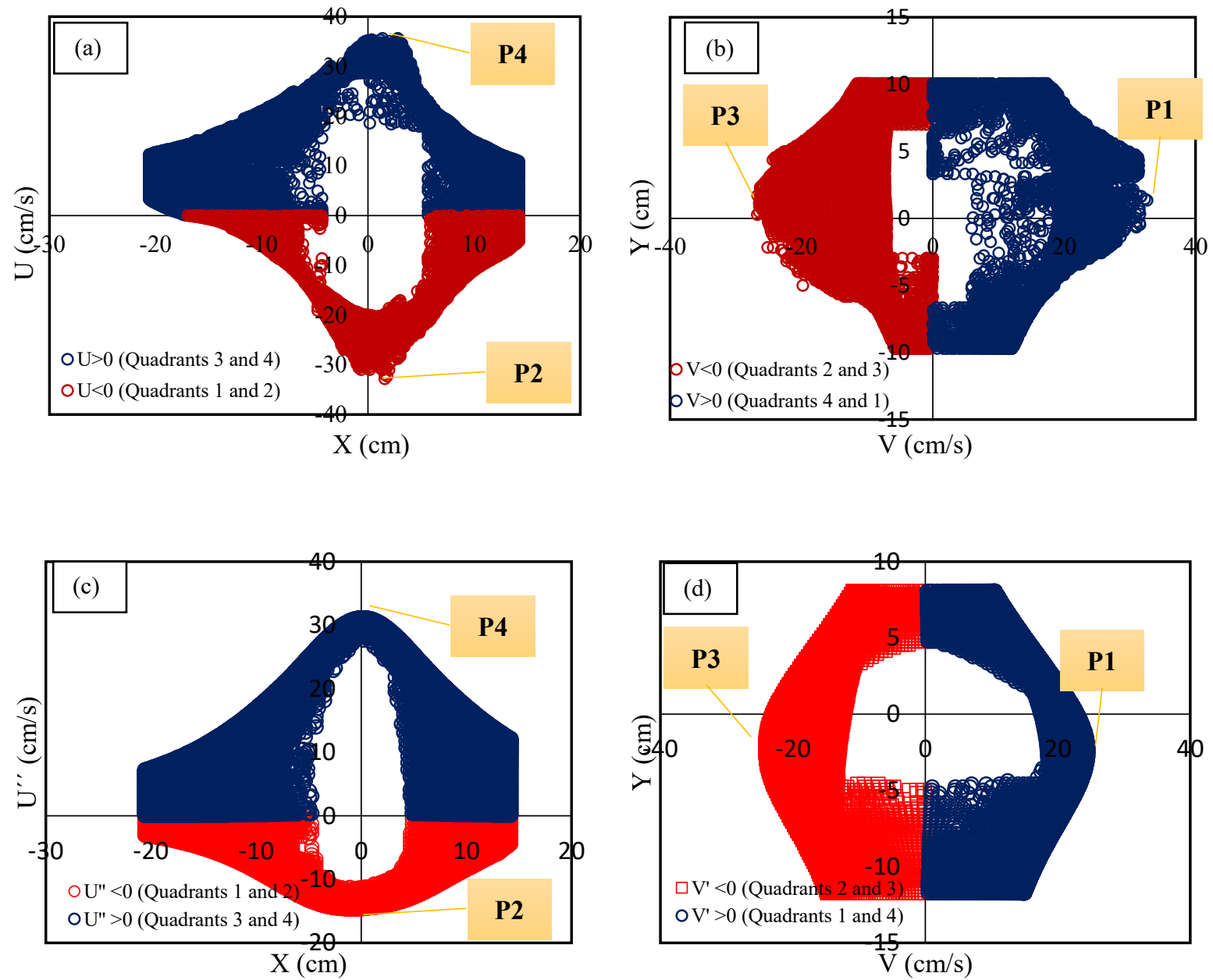

Figure 8- Distribution of $U$ and $V$ against $X$ and $Y$ axes at $Z=0.5 h$ indicating: experimental results (a) $\mathrm{P} 2$ and $\mathrm{P} 4$, (b) $\mathrm{P} 1$ and P3; Burgers/Rott model (c) P2 and P4, (d) P1 and P3.

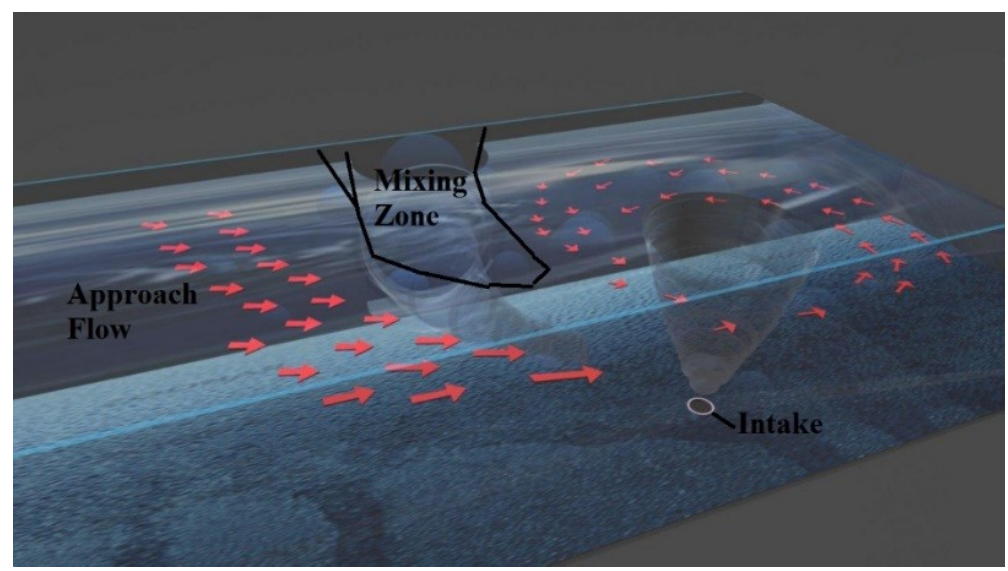

Figure 9-Schematic diagram of vortex, mixing zone and approach flow 
Naderi, V., Farsadizadeh, D., Lin, C. and Gaskin, S.J. (2019) A 3D study of an air core vortex using HSPIV and flow visualization, Arabian Journal for Science and Engineering. doi:10.1007/s13369-019-03764-3
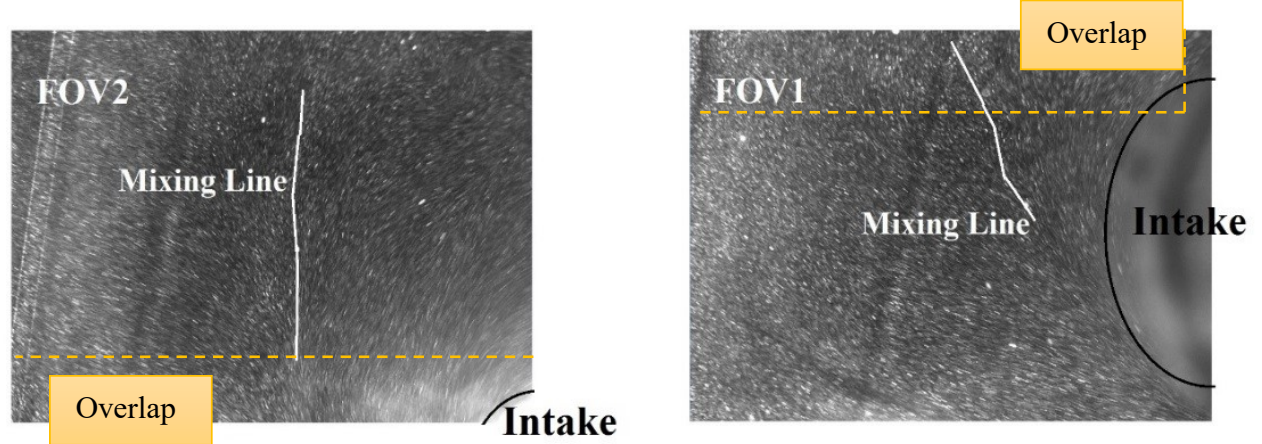

Figure 10-Instantaneous streak-line images for horizontal FOVs at $Z=0.75 \mathrm{~h}$.

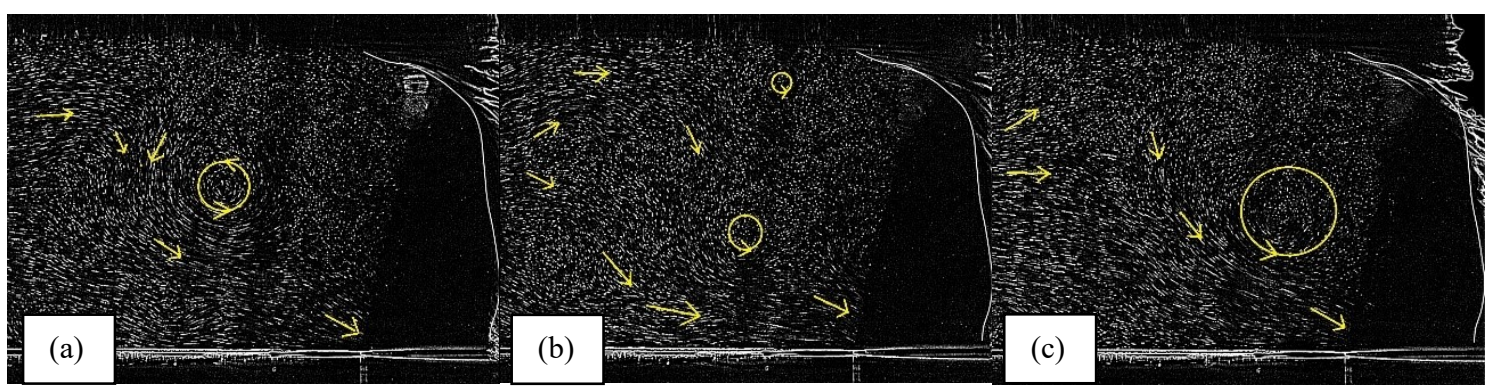

Figure 11- Instantaneous streak-line images for vertical FOVs at $Y=0 \mathrm{~cm}$ : (a) $t=0 \mathrm{~s}$; (b) at $t=$ $4 \mathrm{~s} ;$ (c) at $t=8 \mathrm{~s}$

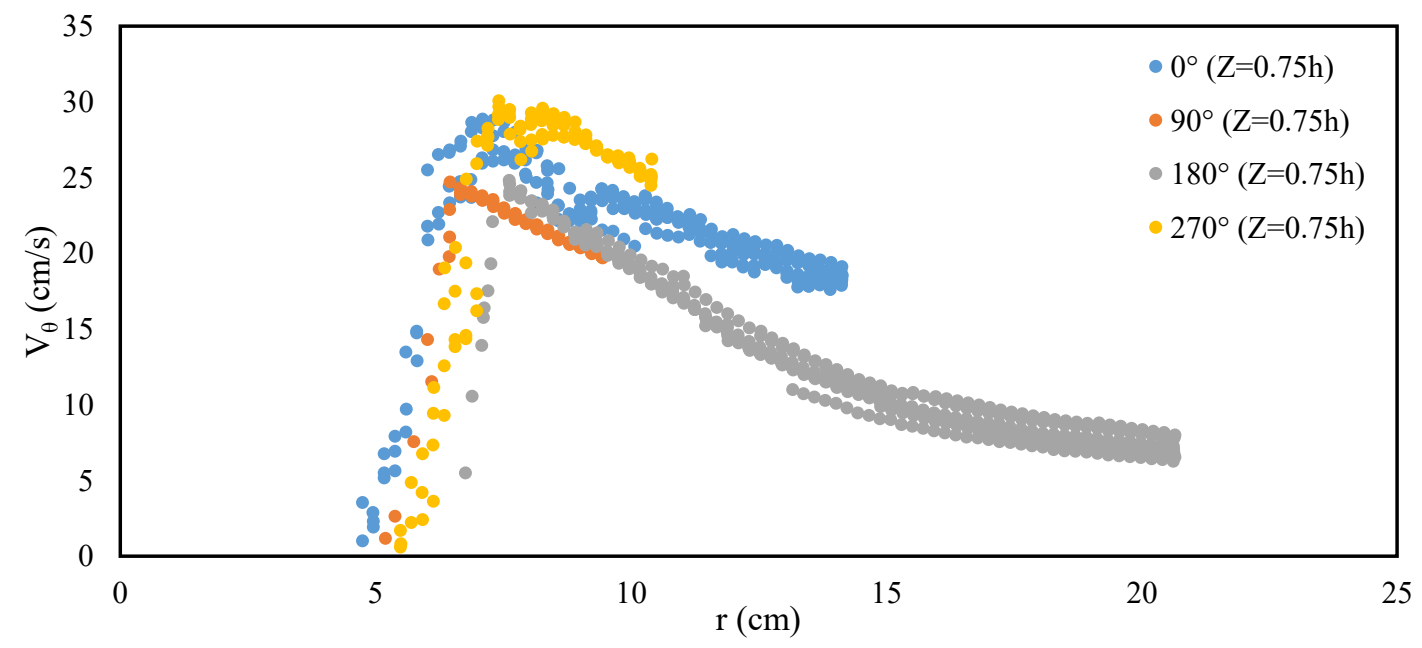

Figure 12 - Tangential velocity distribution of the vortex at $Z=0.75 \mathrm{~h}$ at four cardinal positions $\left(0^{\circ}, 90^{\circ}, 180^{\circ}\right.$, and $270^{\circ}$ at $\pm 2^{\circ}$ at each position). 
Naderi, V., Farsadizadeh, D., Lin, C. and Gaskin, S.J. (2019) A 3D study of an air core vortex using HSPIV and flow visualization, Arabian Journal for Science and Engineering. doi:10.1007/s13369-019-03764-3

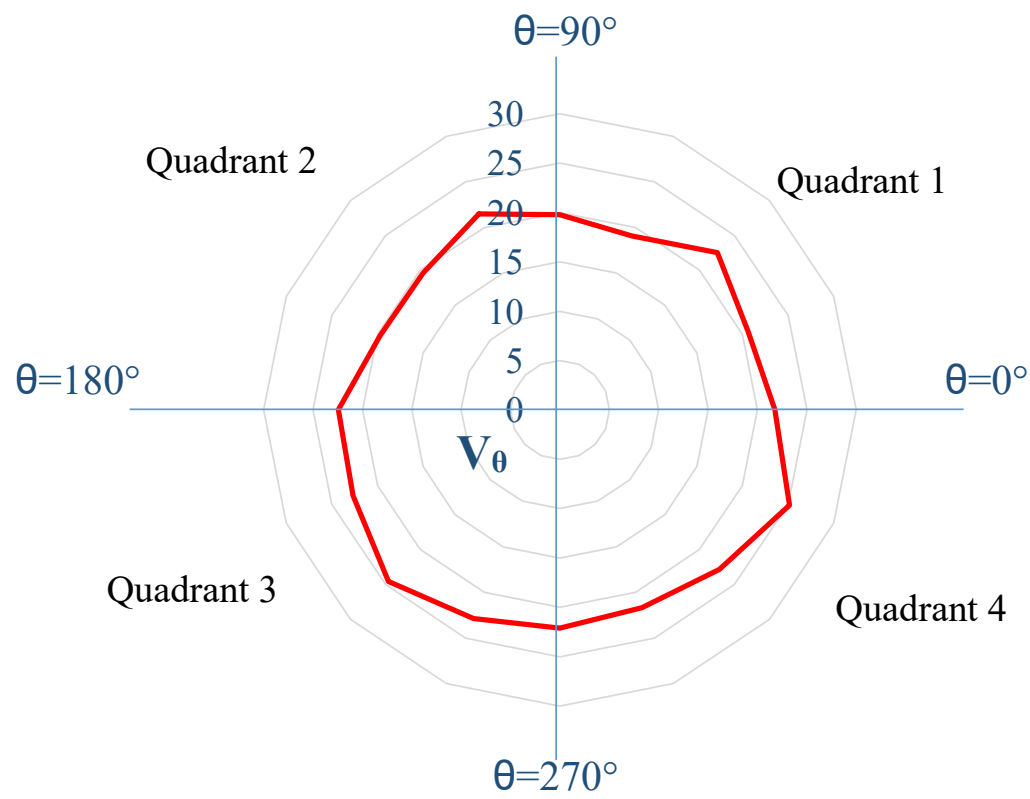

Figure 13-Magnitude of the averaged tangential velocity distribution (between $r=5$ to $10 \mathrm{~cm}$ ) of the vortex at $Z=0.75 h$ at angular intervals of $22.5^{\circ}$ of the vortex flow field.

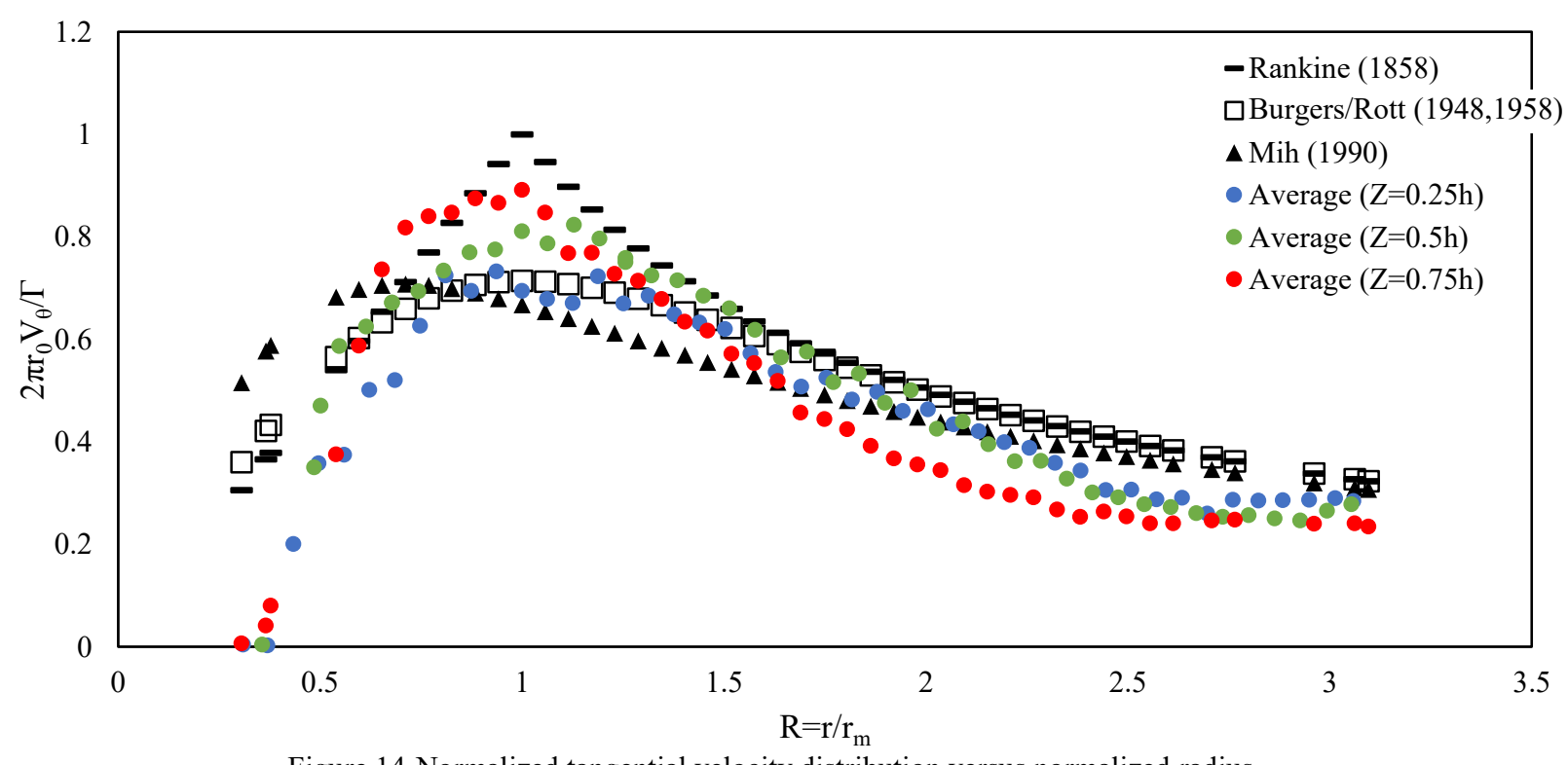

Figure 14-Normalized tangential velocity distribution versus normalized radius 
Naderi, V., Farsadizadeh, D., Lin, C. and Gaskin, S.J. (2019) A 3D study of an air core vortex using HSPIV and flow visualization, Arabian Journal for Science and Engineering. doi:10.1007/s13369-019-03764-3

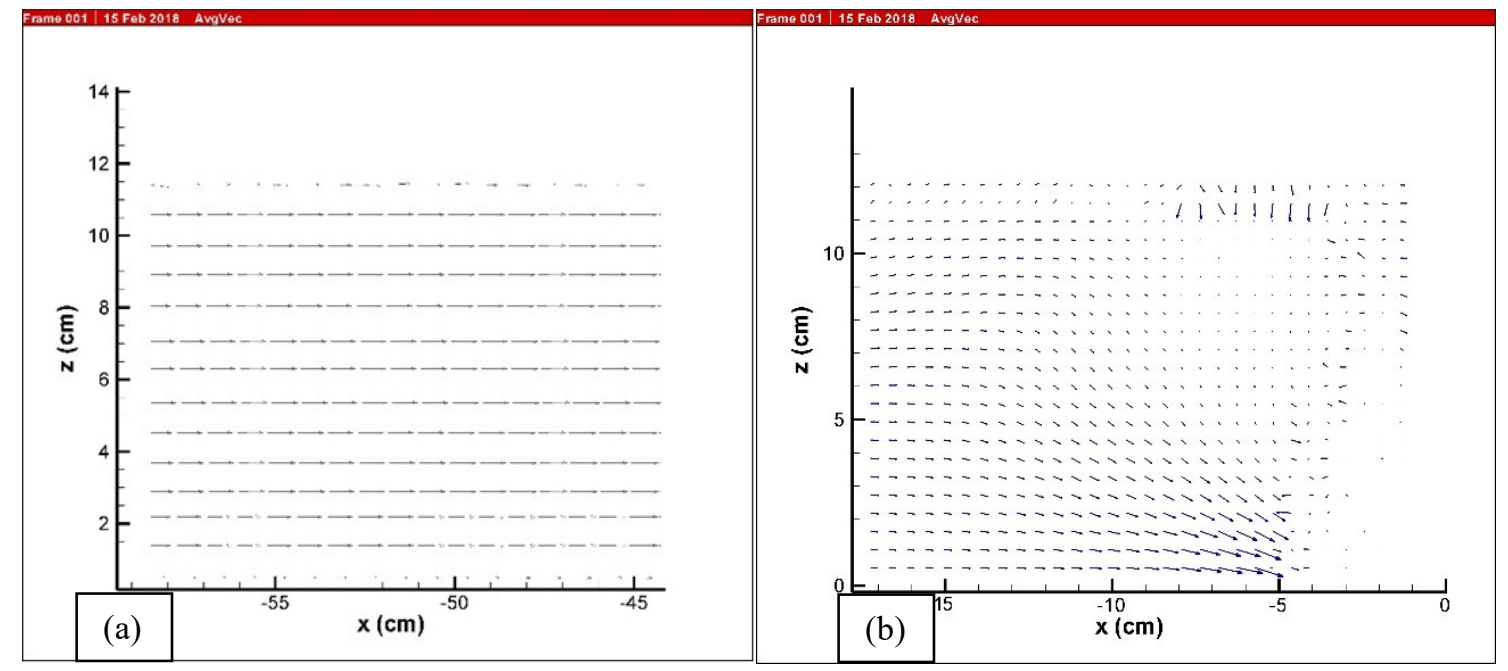

Figure 15-Velocity vector field of vertical PIV at $Y=0 \mathrm{~cm}$ : (a) far upstream from bottom intake; (b) right upstream of the bottom intake (note that the intake origin is at $X=0 \mathrm{~cm}$ and $Z=0 \mathrm{~cm}$ ) 
Naderi, V., Farsadizadeh, D., Lin, C. and Gaskin, S.J. (2019) A 3D study of an air core vortex using HSPIV and flow visualization, Arabian Journal for Science and Engineering. doi:10.1007/s13369-019-03764-3
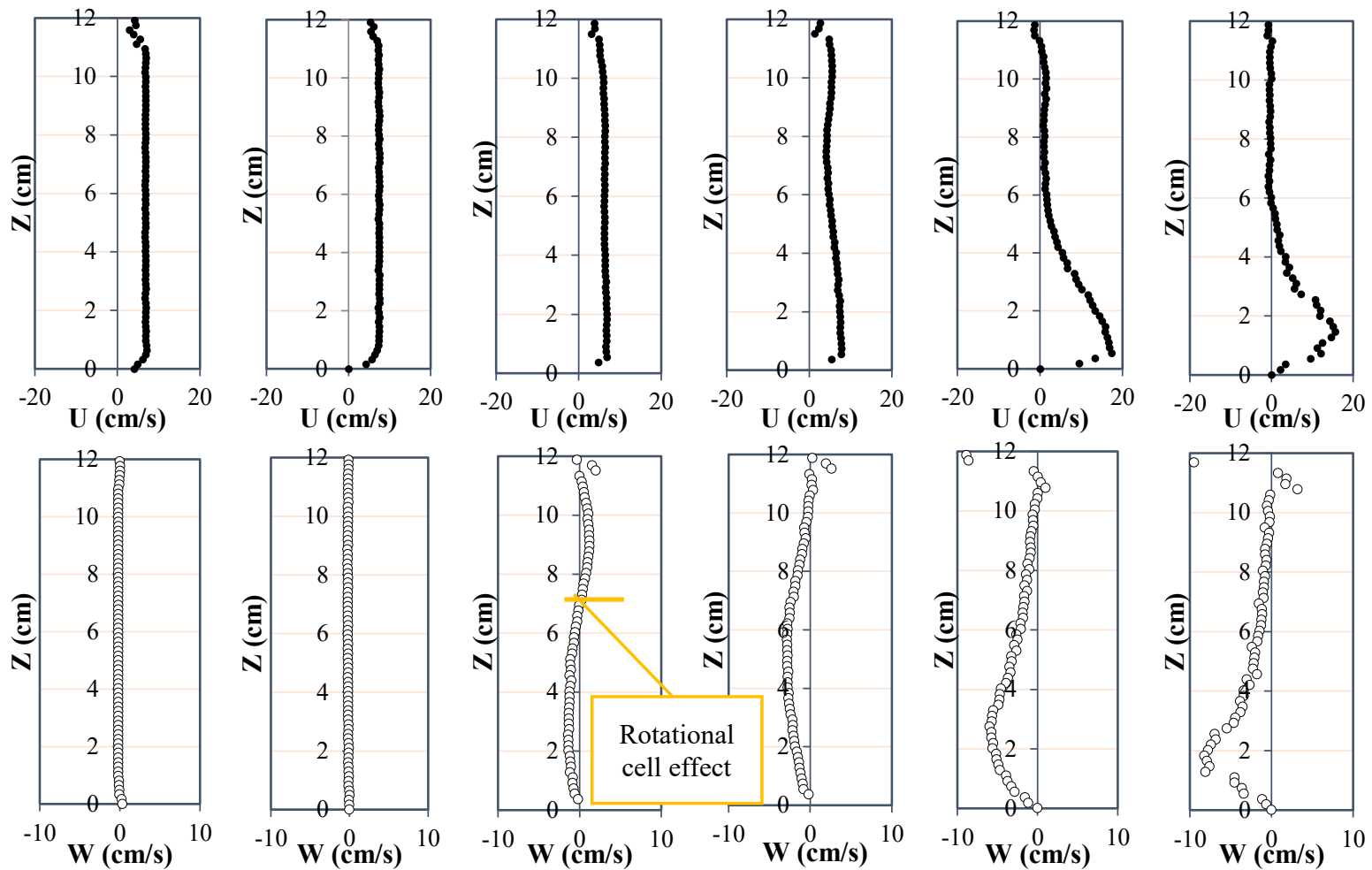

Figure 16- Streamwise $(U)$ and vertical $(W)$ velocity profiles in $Z$-direction at $Y=0 \mathrm{~cm}$.

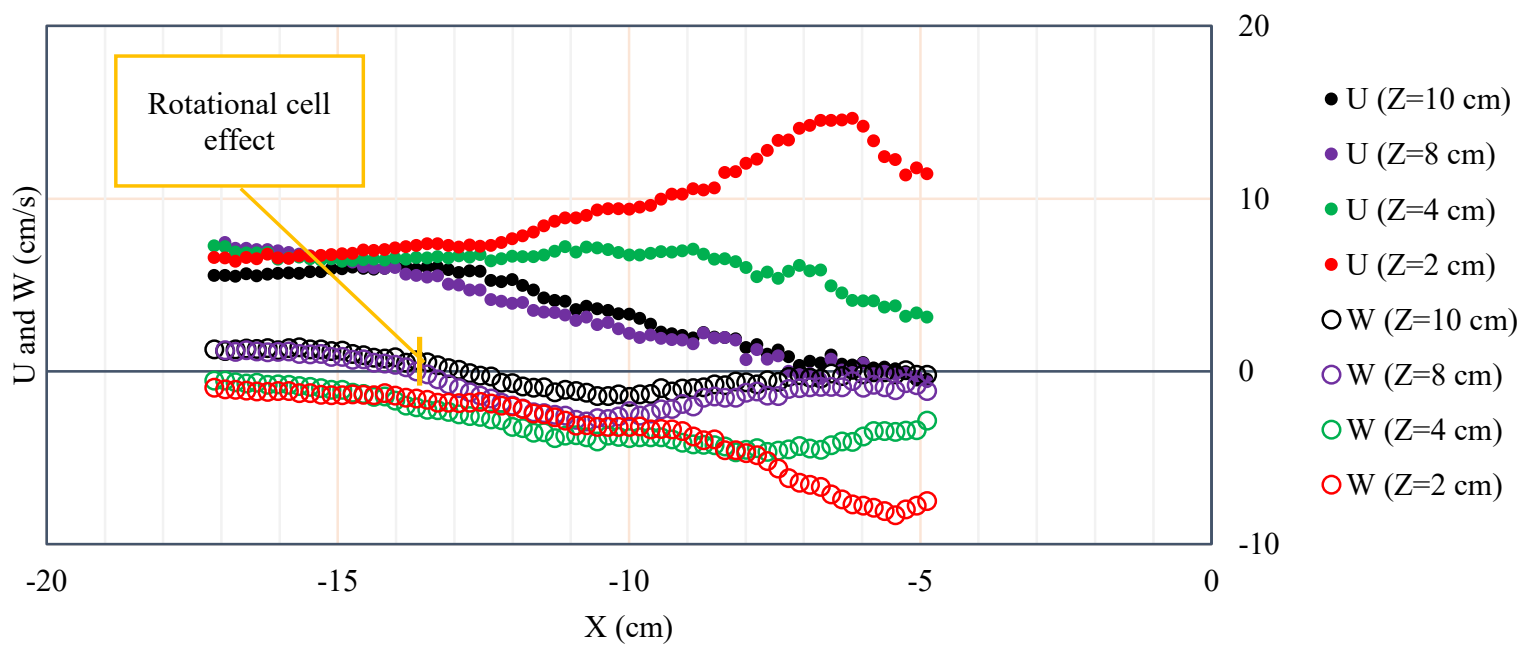

Figure 17- Streamwise $(U)$ and vertical $(W)$ velocity profiles in $X$-direction at $Y=0 \mathrm{~cm}$. 
Naderi, V., Farsadizadeh, D., Lin, C. and Gaskin, S.J. (2019) A 3D study of an air core vortex using HSPIV and flow visualization, Arabian Journal for Science and Engineering. doi:10.1007/s13369-019-03764-3
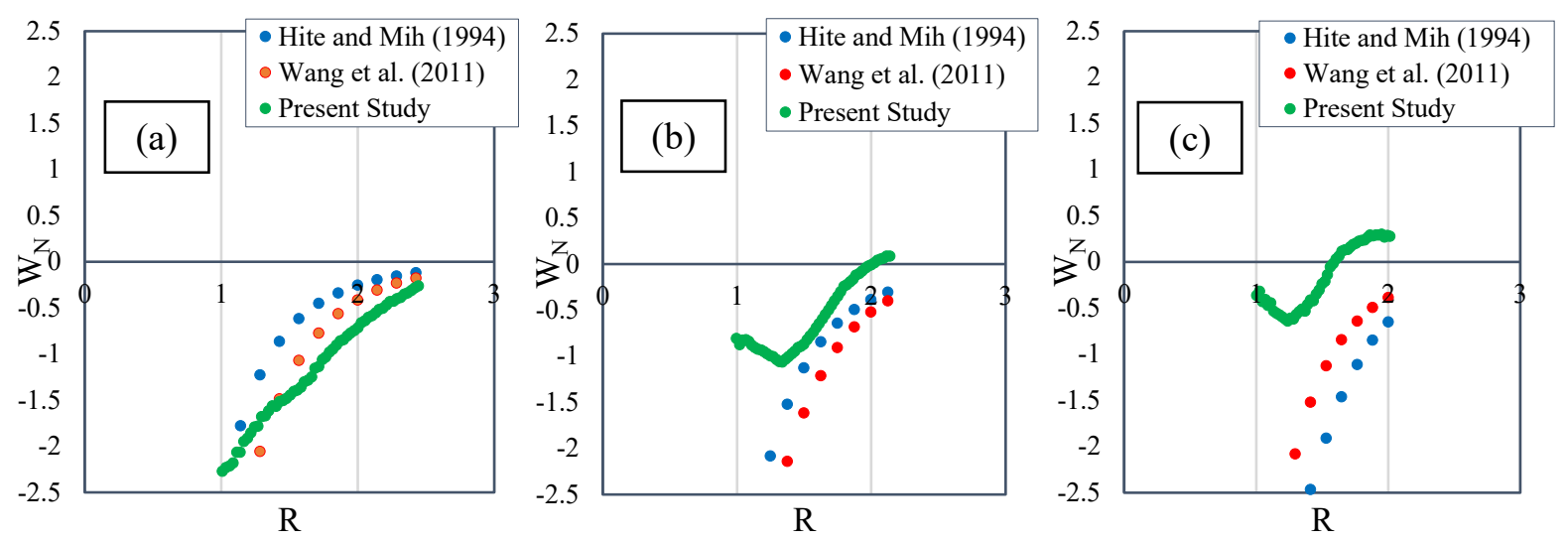

Figure 18-Non-dimensional axial velocity distribution along $R$ at: (a) $Z=0.25 h$; (b) $Z=0.5 h$ and (c) $Z=0.75 h$. 